\title{
Visualization of Flow Field: Application of PLIF Technique
}

\author{
Jiang Bo Peng $\mathbb{D},{ }^{1,2}$ Ze Fang $L v \mathbb{D},{ }^{1,2}$ Xin Yu, ${ }^{1,2}$ Song He Meng, ${ }^{3}$ Hao Dong $\mathbb{D},{ }^{4}$ Fan Deng, ${ }^{5}$ \\ Lin Chen, ${ }^{5}$ De Ying Chen, ${ }^{1,2}$ Rong Wei Fan, ${ }^{1,2}$ Zhi Wei Dong, ${ }^{1,2}$ and Xu Dong Li ${ }^{1,2}$ \\ ${ }^{1}$ National Key Laboratory of Science and Technology on Tunable Laser, Harbin Institute of Technology, Harbin 150080, China \\ ${ }^{2}$ Institute of Opt-Electronics, Harbin Institute of Technology, Harbin 150080, China \\ ${ }^{3}$ Science and Technology on Advanced Composites in Special Environment Laboratory, Harbin Institute of Technology, \\ Harbin 150080, China \\ ${ }^{4}$ College of Aerospace Engineering, Nanjing University of Aeronautics and Astronautics, Nanjing 210016, China \\ ${ }^{5}$ Science and Technology on Space Physics Laboratory, China Academy of Launch Vehicle Technology, Beijing 100078, China
}

Correspondence should be addressed to Jiang Bo Peng; pengiiangbo_2004@126.com

Received 27 September 2017; Accepted 19 November 2017; Published 1 February 2018

Academic Editor: Tingdong Cai

Copyright (C) 2018 Jiang Bo Peng et al. This is an open access article distributed under the Creative Commons Attribution License, which permits unrestricted use, distribution, and reproduction in any medium, provided the original work is properly cited.

\begin{abstract}
The objective of this paper is to apply planar laser-induced fluorescence (PLIF) technology to flow field visualization. This experiment was carried out in a one-meter wind tunnel to study the wake flow field around a circular cylinder. This experiment studied the method of injecting tracer into the flow field; the frequency of the vortex in the wake field and the vortex speed are quantitatively analyzed. This paper gives the correspondence between the speed of the flow field and the frequency of the laser, which could be used as a rough reference standard for future wind tunnel visualization experiments. The result shows that PLIF diagnostic technology has great potential in visualization of flow field.
\end{abstract}

\section{Introduction}

The flow around a circular cylinder is a classic problem of hydrodynamics, knowledge of which is essential for basic understanding and technical applications [1]. In many vehicles such as cars and aircrafts, there are many cylindrical structures on the surface. In the movement of these vehicles, the interaction between the air and the cylinder is extremely complex, which involves the nonlinear process of flow separation, vortex shedding, lift oscillation, and so on. Therefore, it is more important to understand the law of the flow around the blunt body and its wake flow field. The research has a high theoretical value and the practical significance of the safety of industrial equipment.

In the method of flow field structure measurement, noncontact measurement, for the characteristics of not disturbing the structure of the flow field, has high application value. In the noncontact measurement methods, smokewire technique $[2,3]$, particle image velocimetry (PIV) $[4,5]$, and planar laser-induced fluorescence (PLIF) are the most popular methods to realize flow field visualization.
The smoke-wire technique is often used to visualize the flow in the wind tunnel, and the airflow can be observed by evaporating a smoke-generating liquid from a fine wire by electric heating. Smoke-wire technique is mainly applied to lowspeed flow field measurement, about ten meters per second. PIV technology can measure the two-dimensional speed field in the flow field instantaneously, but particle followability is the main problem. The PLIF technique, with impact less of the flow field structure, high temporal and spatial resolution, and other characteristics, has unparalleled advantages.

PLIF imaging is one of the most promising of these techniques because it is species- and quantum state-specific and is therefore sensitive to species composition, temperature, number density, and velocity [6]. In the application of PLIF technology, NASA used NO as a tracer to carry out a large number of experiments $[7,8]$, but $\mathrm{NO}$ as a toxic gas, and chemically active, the experimental conditions are very demanding.

This experiment used acetone as the tracer, as acetone has good signal intensity, low toxicity, high vapor pressure, accessible absorption (225-320 nm), and fluorescence (350- 


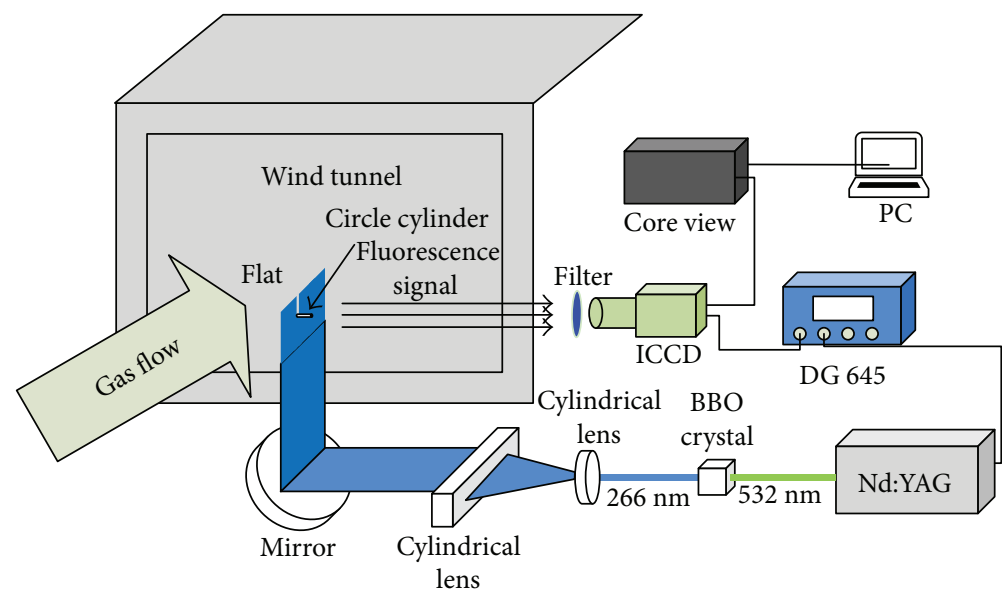

FIGURE 1: Experimental set-up schematic diagram.

$550 \mathrm{~nm}$ ) [9-11]. Since the peak of the acetone fluorescence is near $440 \mathrm{~nm}$, conventional lenses can capture the fluorescence signal. This experiment was carried out at normal pressure and temperature, and the speed of the flow field was low; in the vortex, the temperature and pressure would not change greatly. When acetone was released into the flow field as a tracer, its fluorescence intensity could approximately represent the spatial distribution of acetone density, which represented the vortex structure. A series of flow field images were taken by a high-speed camera to obtain wake vortex structure development. The main problems addressed in this paper are the delivery of acetone vapor and the exploration of applying PLIF to aerodynamics.

\section{Experimental Details}

The experiments were conducted in a one-meter wind tunnel in Nanjing University of Aeronautics and Astronautics. The wind tunnel is a low turbulence, low noise, and low-speed return wind tunnel system, with an open test section of $1.7 \mathrm{~m}$ (length) $\times 1.5 \mathrm{~m}$ (width) $\times 1.0 \mathrm{~m}$ (height), and the test section is open. The speed of the wind tunnel is from $3 \mathrm{~m} / \mathrm{s}$ to $35 \mathrm{~m} / \mathrm{s}$, with the turbulence intensity of $\varepsilon \leq 0.07 \%$, airflow angle of pitching direction $|\Delta \alpha| \leq 0.5^{\circ}$, and airflow angle of yaw direction $|\Delta \beta| \leq 0.5^{\circ}$.

Acetone was excited by $266 \mathrm{~nm}$ laser as a tracer; it was released into the flow field to label the gas stream. The laser beam emitting about $3-4 \mathrm{~mJ} /$ pulse at frequency rate of $100 \mathrm{~Hz}$ or $500 \mathrm{~Hz}$ was formed into a collimated sheet using a combination of cylindrical lenses. Two cylindrical lenses, with focal lengths of $-22 \mathrm{~mm}$ and $240 \mathrm{~mm}$, formed a cylindrical telescope, spreading the laser beam into a collimated, $70 \mathrm{~mm}$ (width) laser sheet. Another cylindrical lens with $100 \mathrm{~mm}$ focal length focused the sheet to about $100 \mu \mathrm{m}$ waist. Acetone vapor was excited by the laser sheet. The fluorescence from part of the illuminated sheet was recorded with an 8-bit intensified CCD camera. The minimum gate width of ICCD was $1 \mathrm{~ns}$. In consideration of the acetone fluorescence lifetime is $8-11 \mathrm{~ns}$, the gate width was set to $20 \mathrm{~ns}$ in this experiment, the camera has a maximum frame rate of $500 \mathrm{~Hz}$ in full format, and the CCD array was $1280 \times 1024$ pixels.

The camera was equipped with a Nikkor AF-S 300mm f/4D IF-ED, whose aperture was $77 \mathrm{~mm}$. A Schott glass filter (WG305) was installed in front of the lens to block $266 \mathrm{~nm}$ scattered light. The camera was interfaced to a PC computer to control the camera and to record the images. The ICCD camera imaged a $112.5 \times 90 \mathrm{~mm}^{2}$ area of the flow field, giving a spatial resolution of about $88 \mu \mathrm{m}$ per pixel. The PLIF system timing is controlled by DG645 (Stanford Research Systems).

Figure 1 shows a schematic representation of the experimental set-up. Liquid acetone was stored in a 1.0-liter preheated tank. A certain amount of gaseous acetone and nitrogen as dilution gas flew into the mixing tank, respectively. The molar fraction of acetone in the mixed gas was fixed at $18 \%$. The exit of the acetone tank was connected to a stainless flatbed, controlled by a gas flow meter in order to guarantee a certain flow rate. The mixed gas was injected from a slot and was diluted into the wind tunnel flow field.

In the experiments, the circular cylinder's diameter was $2 \mathrm{~mm}$. The distance between the slot and the cylinder was $5.9 \mathrm{~cm}$. Laser sheet paralleled to the flat, and the distance between the flat and the laser sheet was from $1 \mathrm{~mm}$ to $4.5 \mathrm{~mm}$. The Reynolds number was from 70 to 800 . The jet direction of the slot was at an angle of $45^{\circ}$ and $90^{\circ}$ to the surface of the flat. It should be noticed that all measurements presented here were performed with $\mathrm{N}_{2}$ as carrier gas. In the experiments, we investigated the effect of acetone injection angle and the distance between the laser sheet and the flat on the PLIF image. A series of flow field images were taken by a high-speed camera to obtain wake vortex structure development. Depending on a special kind of vortex structure, the vortex's speed and generation frequency are calculated.

\section{Experimental Results}

This section presents PLIF visualization results of a circular cylinder wake flow field at various experimental conditions. 

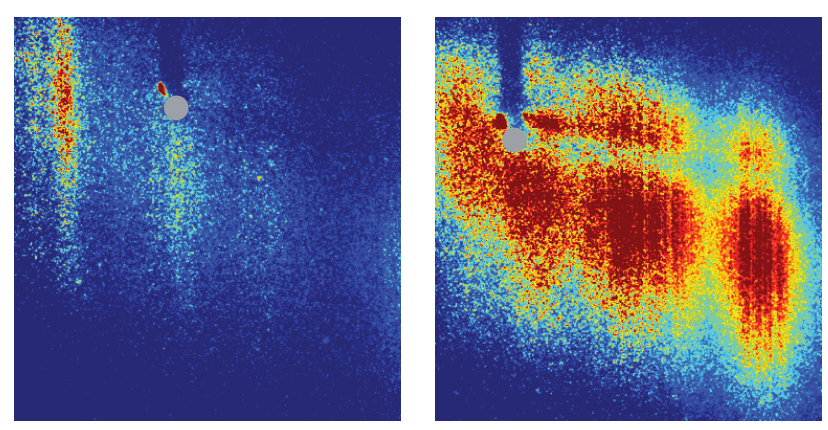

(a)

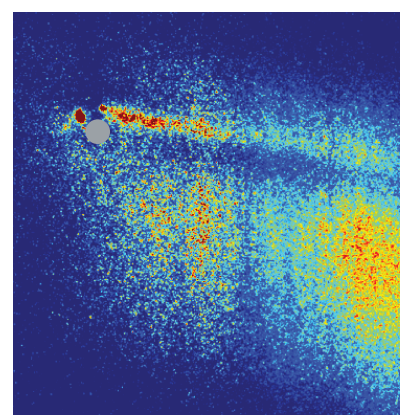

(c) (b)

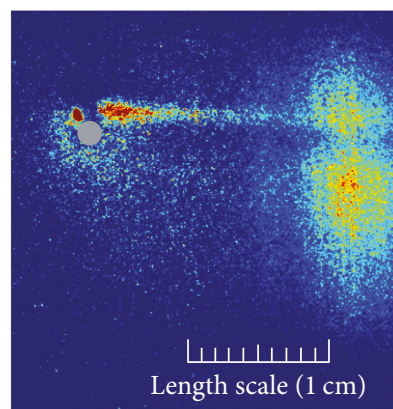

(d)

Figure 2: Spanwise scan of laser sheet. The diameter $=2 \mathrm{~mm}$, sheet positions were $1,2.5,4$, and $4.5 \mathrm{~mm}$ from the flat, Reynolds number $=70$, and frame rate $=100 \mathrm{~Hz}$. The jet direction was at an angle of $90^{\circ}$ to the surface of the flat.

3.1. PILF Images of Wake Flow Field Depend on Laser Sheet Position. The flow field structures at different distances from the surface of the flat plate were shown in Figure 2. In Figure 2(a), the distance between the sheet and the flat was $1 \mathrm{~mm}$. Because the flat has a viscous force on the flow, the flow speed near the flat was far slower than the average speed, and the relative molecular mass of mixed gas (acetone vapor and $\mathrm{N}_{2}$ ) was 33.43 , heavier than air, acetone in the flow field obliquely rightward flew. As the distance from the sheet is bigger, the flow speed got faster. The direction of the acetone tends to be more horizontal, shown in Figures 2(b)-2(d). This means that when the speed of the flow field is fast enough, the followability of the acetone gas mixture can be ignored. In Figure 2(b), the fluorescence intensity was far stronger than others; it indicated that after the acetone flew out of the slot, it was concentrated about $2.5 \mathrm{~mm}$ from the flat in the flow field in this condition. The best position of the sheet in the experiment is related to many factors; two of the main factors are the injection speed of the mixed gas and the speed of the flow field.

\subsection{PILF Images of Wake Flow Field Depend on Different Jet} Directions. Figure 3 shows wake flow field images that depend on jet directions. In Figure 3(a), the jet direction was $90^{\circ}$ to the surface of the flat. The acetone had an initial injection speed. After jetted from the slot, the acetone had not been able to mix well into the wake field where the sheet was located. This is the main cause of the vague image. In Figure $3(\mathrm{~b})$, the jet direction was $45^{\circ}$ to the surface of the flat. Even though the initial injection speed was the same,

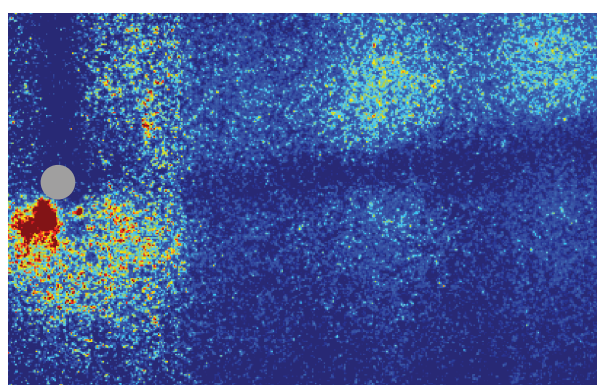

(a)

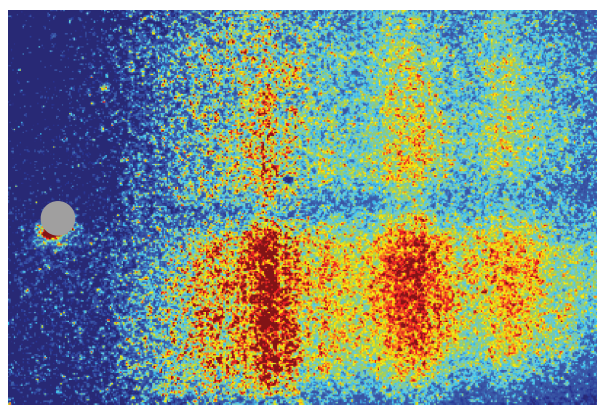

(b)

FIgURe 3: Wake flow fields depend on different jet directions. (a) The jet direction was at an angle of $45^{\circ}$ to the surface of the flat and (b) was $90^{\circ}$. The diameter $=2 \mathrm{~mm}$, Reynolds number $=100$, and sheet position $=2 \mathrm{~mm}$.

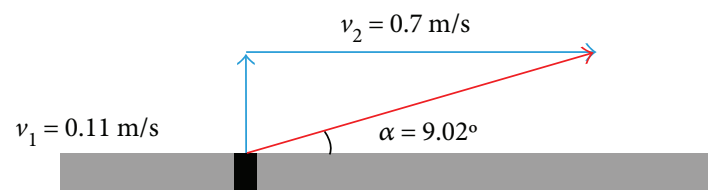

(a)

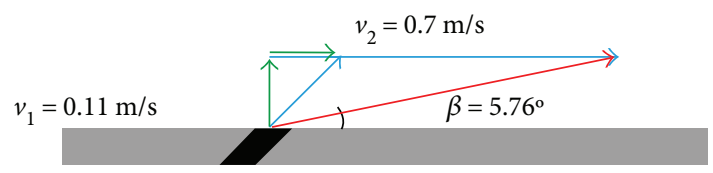

(b)

Figure 4: Effect of gas injection direction on tracer flow direction. (a) The jet direction was at an angle of $45^{\circ}$ to the surface of the flat, and (b) was $90^{\circ}$. Flow speed $\left(v_{1}\right)=0.7 \mathrm{~m} / \mathrm{s}$, and mixed gas outlet speed $\left(v_{2}\right)=0.11 \mathrm{~m} / \mathrm{s}$.

the injection direction was more conducive to acetone mixing into the sheet flat. Figure 4 shows the effect of the gas injection direction on the flow of the mixed gas. In Figure $4(\mathrm{a})$, the jet direction was $90^{\circ}$. The angle between the tracer speed and the plane was $9.02^{\circ}$. And when the gas was ejected from the slot, there was no initial speed in the direction of the flow field.

In Figure 4(b), the jet direction was $45^{\circ}$. The angle between the tracer speed and the plane was $5.76^{\circ}$. The initial speed in the flow direction (the horizontal green arrow) was about $0.08 \mathrm{~m} / \mathrm{s}$, faster than Figure 4(a); the initial speed vertical to the flow direction (the vertical green arrow) was $0.08 \mathrm{~m} / \mathrm{s}$, slower than Figure 4(a). This means that when the gas is jetted at a direction of $45^{\circ}$, the tracer has a better 


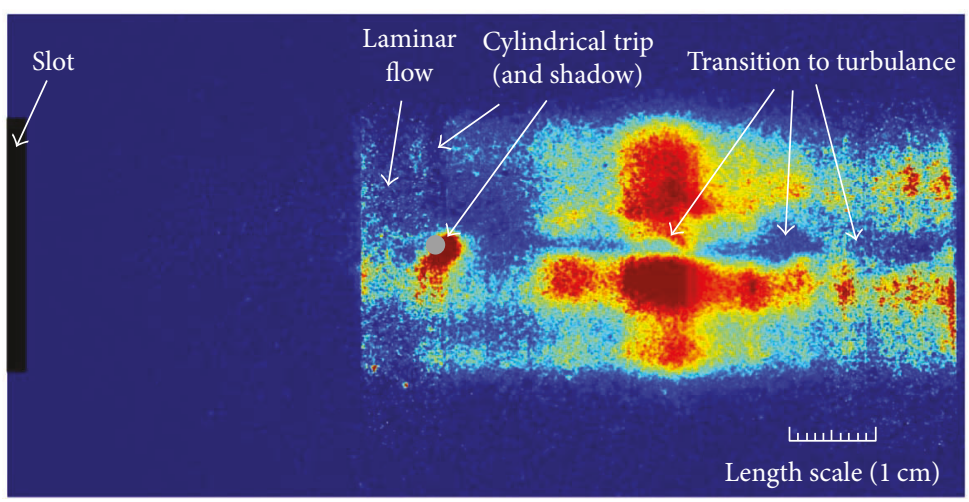

FIgure 5: The typical $100 \mathrm{~Hz}$ acetone PLIF image. The diameter $=2 \mathrm{~mm}$; the distance between the cylinder and the slot was $5.9 \mathrm{~cm}$; the distance between flat and laser sheet was $3 \mathrm{~mm}$; Reynolds number $=800$; and frame rate $=500 \mathrm{~Hz}$.

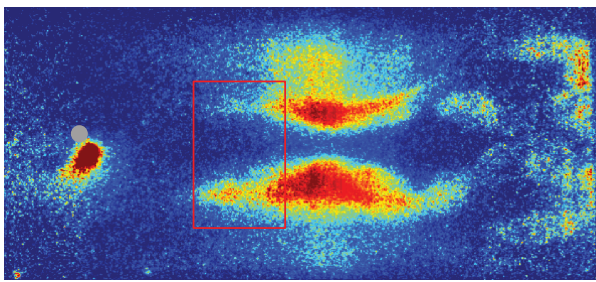

(a)

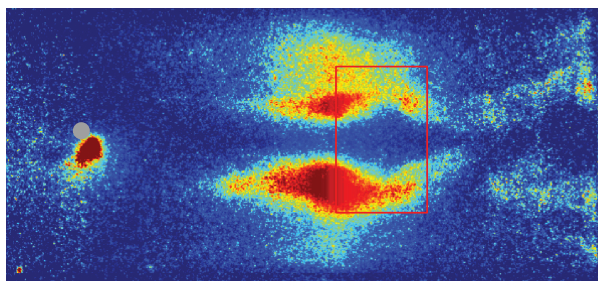

(c)

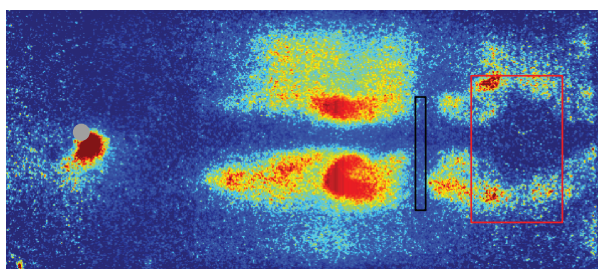

(e)

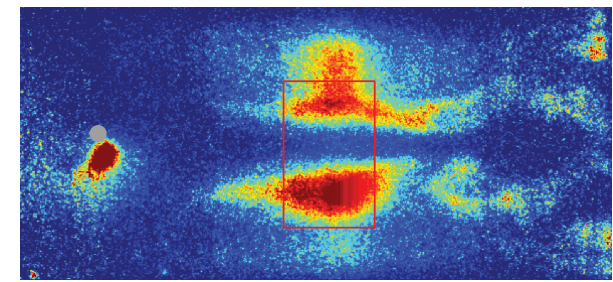

(b)

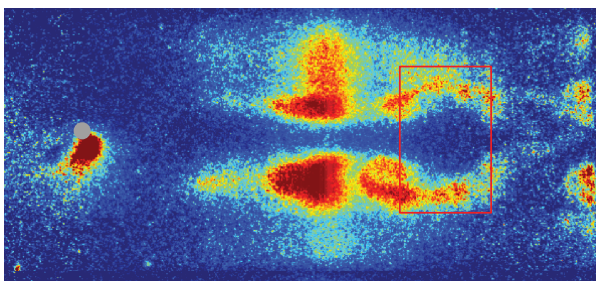

(d)

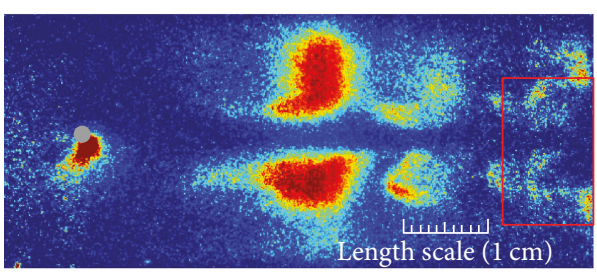

(f)

FIGURE 6: Wake vortex structure development in flow field. Diameter $=2 \mathrm{~mm}$, Reynolds number $=500$, and sheet position $=3 \mathrm{~mm}$. The distance between the cylinder and the slot was $5.9 \mathrm{~cm}$. The jet direction was at an angle of $45^{\circ}$ to the surface of the flat. The frame rate was $500 \mathrm{~Hz}$.

followability, and the tracer can be better adhering to the surface of the plate, reflecting the surface flow field structure. In the later part of this paper, the direction of mixed gas injection was $45^{\circ}$ to the surface of the flat.

\subsection{PLIF Images of Flow Field Transition from Laminar to} Turbulent Flow. In Figure 5, the circular cylinder shows the position of the cylinder's bottom side, because the cylinder was not vertical to the flat in the camera's perspective, the cylinder's position did not coincide with the center of the fluorescent signal. The flow was left-to-right and vertical to the ground. Between the slot and the cylinder, the flow was mostly smooth and laminar. No irregular flow structures were observed upstream of the cylinder. The flow labeled with acetone did not show a visible change in the width before and after passing by the cylinder. The wake flow behind the cylinder showed some visible streaks that did not show up in the upstream flow. These streaks are evidence of perturbations induced by the cylinder and may be associated with streamwise vorticity. The streaks within the roughness wake soon become unstable, oscillating with periodic structures and then breaking down into much more irregular structures. In the diagram, this region of the flow is labeled "transition to turbulence." 


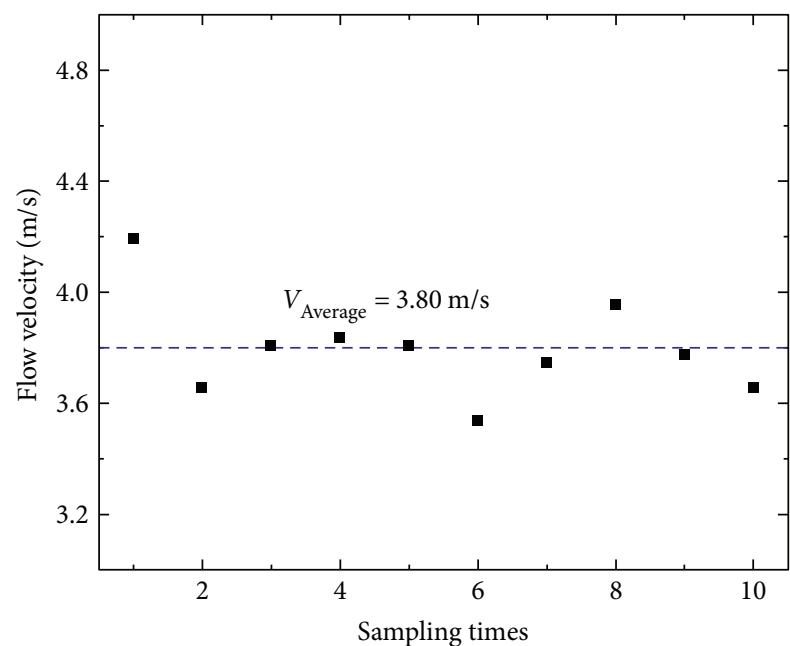

Figure 7: The speed of the wake flow in the sample. Reynolds number $=800$, the diameter $=2 \mathrm{~mm}$, the sheet position $=3 \mathrm{~mm}$, and the distance between the cylinder and the slot was $5.9 \mathrm{~cm}$. The jet direction was at an angle of $45^{\circ}$ to the surface of the flat.

We can get a clear wake field structure image by the PLIF visualization technology, which provides both highquality wake vortex structure picture and visualization of the formation. Some basic features will be discussed in the next section.

3.4. Wake Vortex Structure Development. Figure 6 is a series of continuous of the wake vortex, which showed the formation and development of wake vortexes. The acetone carried by the air showed the changes of the flow field and vortex generation and shedding. Because the vortex generation and shedding was quasiperiodic, the distance between each adjacent vortex was approximately equal. As we mentioned in the front of this paper that each pixel represents $119 \mu \mathrm{m}$ actually, the distance between each adjacent vortex can be reversed by the pictures. As the frequency of taking those pictures was known, the frequency of each vortex generation can be calculated.

The average speed of the wake vortex is estimated by measuring the speed of the special structure in the wake field, like the black gap shown in Figure 6. Figure 7 shows the speed and average speed of 10 samples. The average speed was $3.80 \mathrm{~m} / \mathrm{s}$, and the RSD (relative standard deviation) was $4.7 \%$. In two adjacent cycles, this gap would appear twice in a particular area. But there are some slight deviations from the two appearances that need to be corrected by the average speed. Figure 8 shows the period and average period time of 20 samples. The average period was $5.07 \mathrm{~ms}$. The RSD was $8.0 \%$. The frequency of the vortex generation was about $197 \mathrm{~Hz}$. The distance of every adjacent vortex was approximately $19.27 \mathrm{~mm}$.

In this experiment, the maximum frequency of the laser is $500 \mathrm{~Hz}$. The maximum stable wind speed produced by the one-meter wind tunnel is about $21 \mathrm{~m} / \mathrm{s}$. In order to be able to calculate the speed of the vortex, one vortex need to be captured by two adjacent photos. In this experiment, the maximum wind speed that satisfies the above

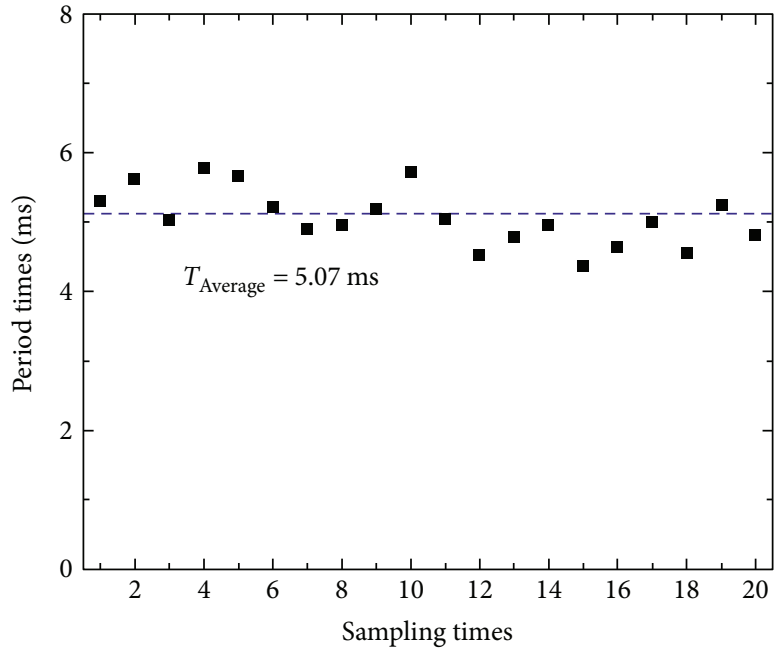

Figure 8: The period of the wake flow in the sample. Reynolds number $=800$, the diameter $=2 \mathrm{~mm}$, the sheet position $=3 \mathrm{~mm}$, and the distance between the cylinder and the slot was $5.9 \mathrm{~cm}$. The jet direction was at an angle of $45^{\circ}$ to the surface of the flat.

postulate is about $18 \mathrm{~m} / \mathrm{s}$. If this standard is used, if measuring the wake flow field in the supersonic wind tunnel, the frequency of the laser is at least $10 \mathrm{kHz}$. If the flow speed reaches $10 \mathrm{Mach}$, the laser frequency should be more than $100 \mathrm{kHz}$. The abovementioned laser frequency is the lowest frequency required for the corresponding experimental conditions. If the experiment need to be more accurate, a higher frequency laser is required.

\section{Conclusion}

The flow field around a circular cylinder has been investigated experimentally in a fully developed one-meter wind tunnel. PLIF is the basic measurement technique to diagnose the wake flow field of the cylinder. Acetone was used as the tracer to label the flow field, the $266 \mathrm{~nm}$ laser beam was shaped into a specific size sheet beam by the beam shaping system, which excited the tracer, and the fluorescence signal was captured by a high acquisition frequency ICCD camera and recorded as PLIF image.

In this experiment, some basic problems on applying the PLIF technology to the flow field visualization are discussed: firstly, acetone as the tracer, with its good signal intensity, low toxicity, high vapor pressure, accessible absorption $(225-320 \mathrm{~nm})$, and fluorescence $(350-550 \mathrm{~nm})$, is very suitable for the experimental study of flow field visualization. Secondly, in the acetone vapor injection to the flow field, the injection speed, angle, and other related factors need to be considered, in order to achieve the best experimental results. Thirdly, this paper gives the correspondence between the speed of the flow field and the frequency of the laser, which could be used as a rough reference standard for future wind tunnel visualization experiments.

In further research, the main direction is to improve the laser repetition frequency and camera resolution, in order to achieve higher temporal and spatial resolution. 


\section{Conflicts of Interest}

The authors declare that there is no conflict of interest regarding the publication of this paper.

\section{Acknowledgments}

This work was supported by the National Natural Science Foundation of China (Grant nos. 61405048, 91441130, and 51536002), Fundamental Research Funds for the Central Universities (Grant no. HIT. NSRIF. 2014045), and the National Key Scientific Instrument and Equipment Development Projects of China (no. 2012YQ040164).

\section{References}

[1] S. Günter, "On the force fluctuations acting on a circular cylinder in crossflow from subcritical up to transcritical Reynolds numbers," Journal of Fluid Mechanics, vol. 133, pp. 265-285, 1983.

[2] S. A. Kazemi, M. Nili-Ahmadabadi, A. Sedaghat, and M. Saghafian, "Aerodynamic performance of a circulating airfoil section for Magnus systems via numerical simulation and flow visualization," Energy, vol. 104, pp. 1-15, 2016.

[3] S. M. Batill and T. J. Mueller, "Visualization of transition in the flow over an airfoil using the smoke-wire technique," AIAA Journal, vol. 19, no. 3, pp. 340-345, 1981.

[4] G. Kirkil and G. Constantinescu, "Effects of cylinder Reynolds number on the turbulent horseshoe vortex system and near wake of a surface-mounted circular cylinder," Physics of Fluids, vol. 27, no. 7, article 075102, 2015.

[5] I. Fujita, M. Muste, and A. Kruger, "Large-scale particle image velocimetry for flow analysis in hydraulic engineering applications," Journal of Hydraulic Research, vol. 36, no. 3, pp. 397-414, 1998.

[6] F. Grisch and M. Orain, "Role of planar laser-induced fluorescence in combustion research," AerospaceLab, pp. 1-14, 2009.

[7] P. M. Danehy, C. B. Ivey, B. F. Bathel et al., "Orbiter BLT flight experiment wind tunnel simulations: nearfield flow imaging and surface thermography," in 48th AIAA Aerospace Sciences Meeting Including the New Horizons Forum and Aerospace Exposition, vol. 1571, Orlando, Florida, 2010.

[8] P. M. Danehy, A. P. Garcia, S. E. Borg et al., Fluorescence Visualization of Hypersonic Flow Past Triangular and Rectangular Boundary-Layer Trips, 2007.

[9] M. C. Thurber, Acetone Laser-Induced Fluorescence for Temperature and Multiparameter Imaging in Gaseous Flows, 1999.

[10] M. C. Thurber, F. Grisch, and R. K. Hanson, "Temperature imaging with single-and dual-wavelength acetone planar laser-induced fluorescence," Optics Letters, vol. 22, no. 4, pp. 251-253, 1997.

[11] M. C. Thurber and R. K. Hanson, "Simultaneous imaging of temperature and mole fraction using acetone planar laser-induced fluorescence," Experiments in Fluids, vol. 30, no. 1, pp. 93-101, 2001. 

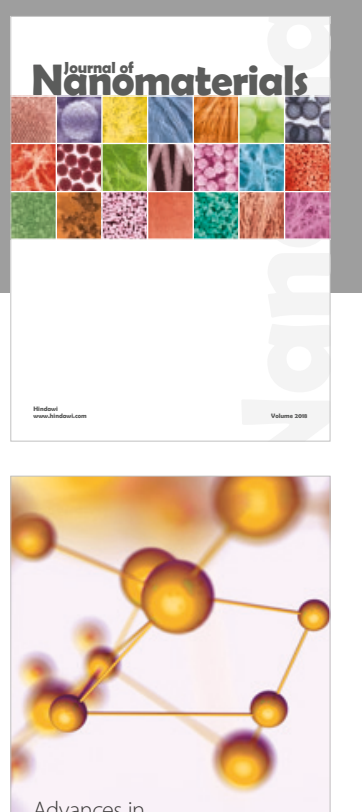

Physical Chemistry
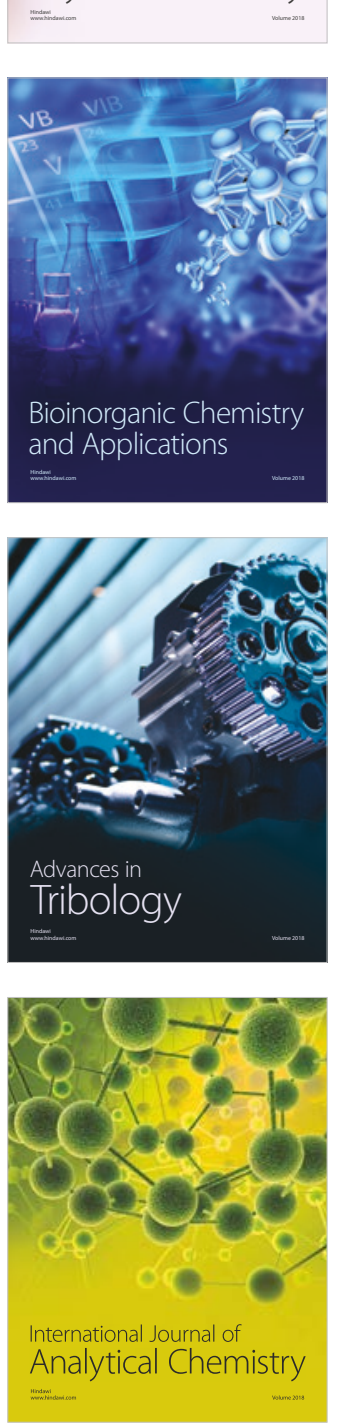

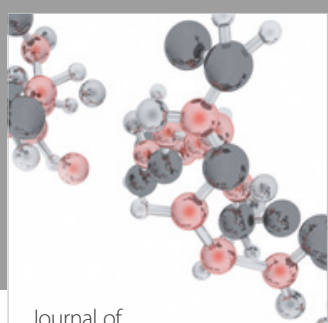

Analytical Methods

in Chemistry

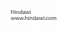

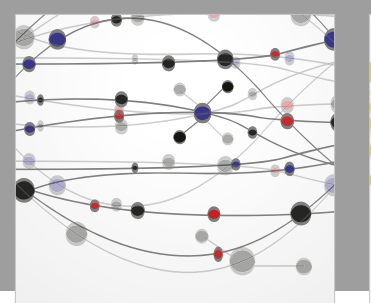

The Scientific World Journal

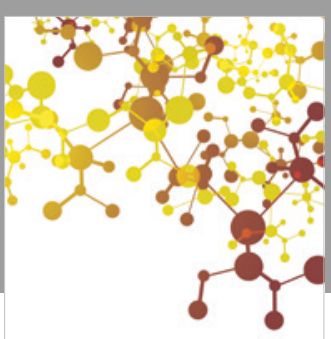

Journal of

Applied Chemistry
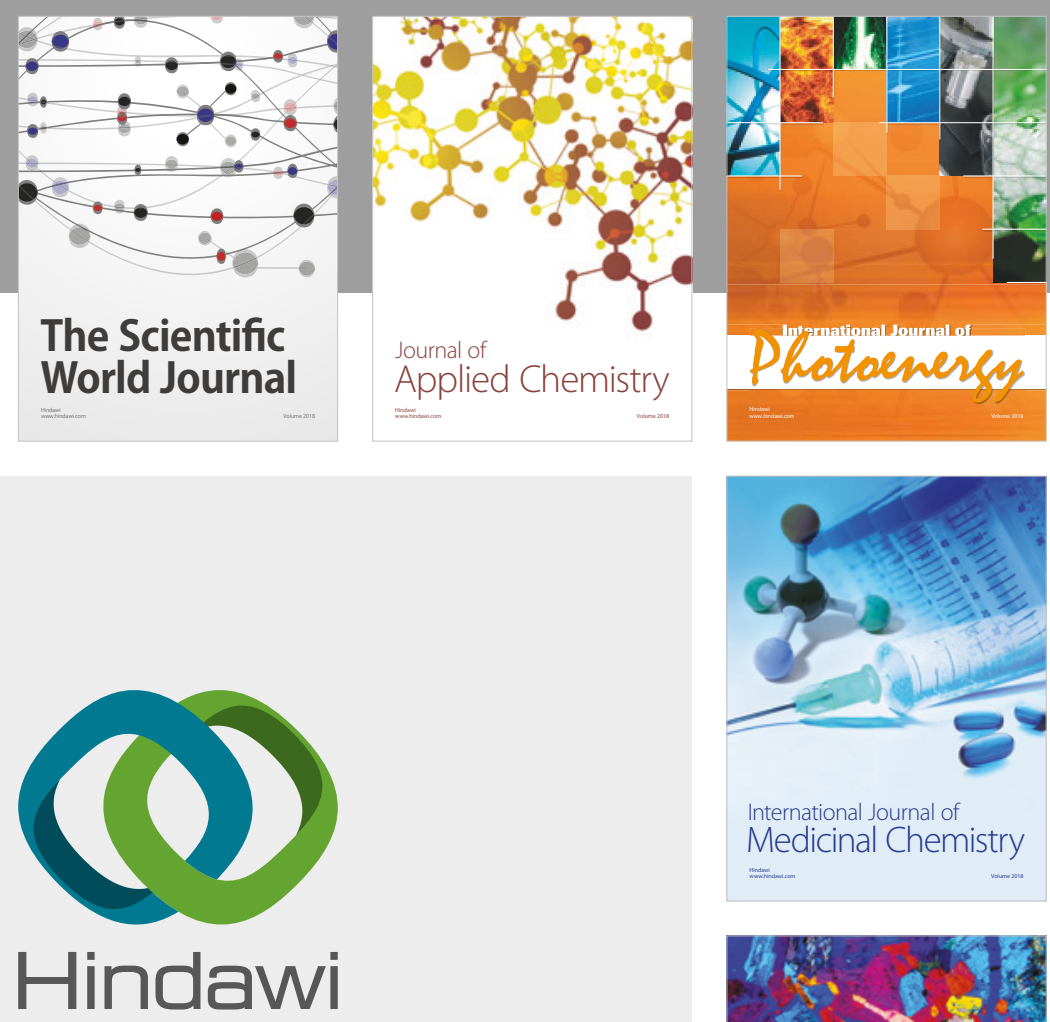

Submit your manuscripts at

www.hindawi.com
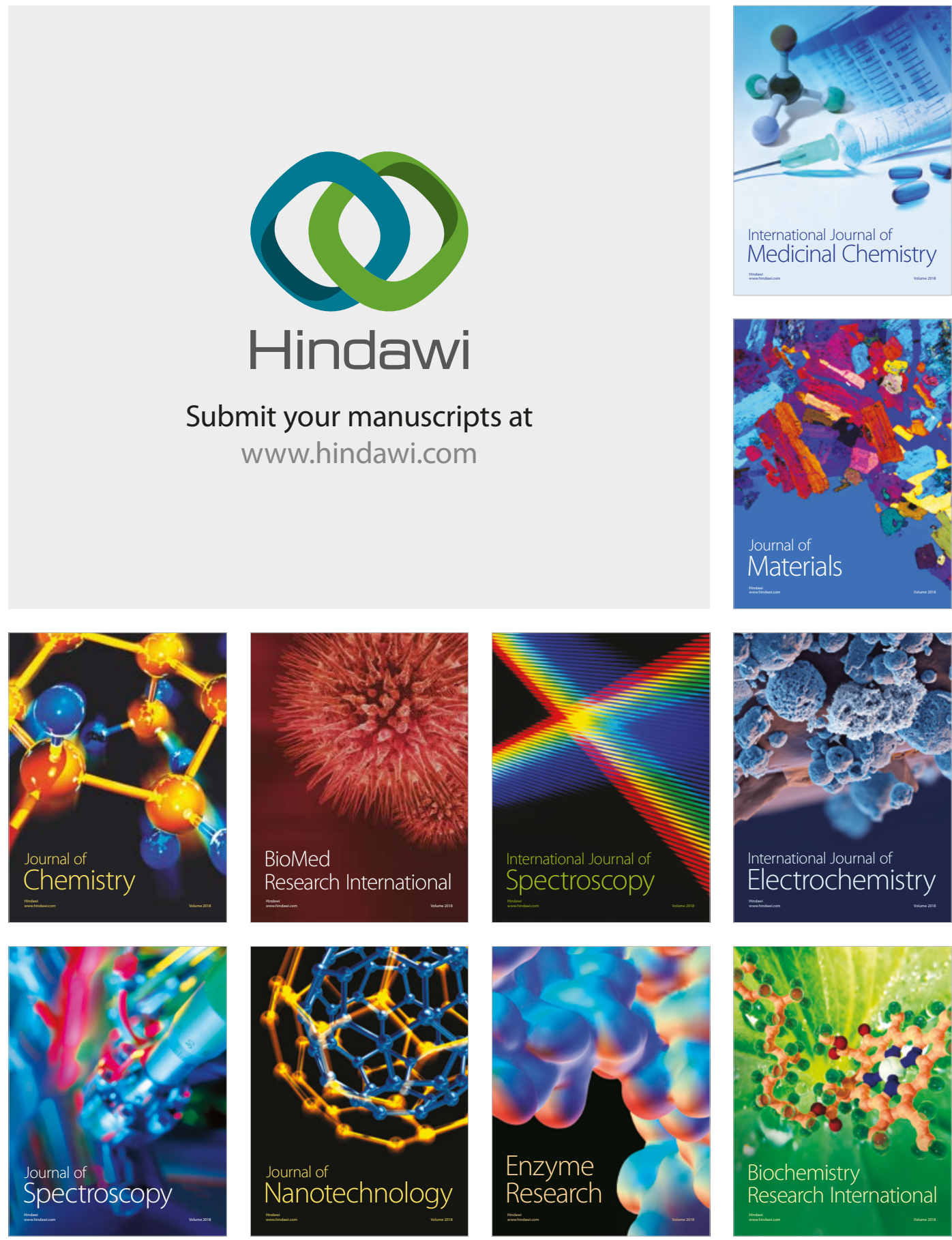
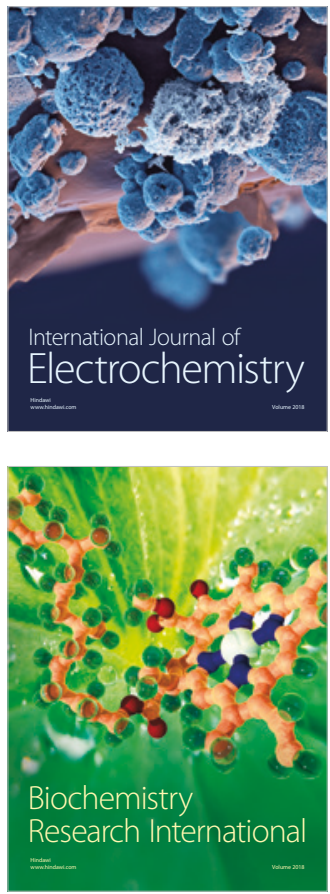